\title{
The Modeling Framework for Through-Metal-Wall Ultrasonic Power Transmission Channels Based on Piezoelectric Transducers
}

\author{
DingXin Yang $(\mathbb{D}$, BaoJian Hou, and Dong Tian \\ Laboratory of Science and Technology on Integrated Logistics Support, National University of Defense Technology, \\ Changsha 410073, China \\ Correspondence should be addressed to DingXin Yang; yangdingxincn@163.com
}

Received 20 January 2019; Accepted 16 April 2019; Published 9 June 2019

Academic Editor: Mitsuhiro Okayasu

Copyright (C) 2019 DingXin Yang et al. This is an open access article distributed under the Creative Commons Attribution License, which permits unrestricted use, distribution, and reproduction in any medium, provided the original work is properly cited.

\begin{abstract}
The investigation of the wireless ultrasonic energy and signal transmission through metal barrier into hermitical metallic structures has become an interesting field of research. The most often constructed acoustic-electric ultrasonic power transmission channels are based on piezoelectric transducers. Effective modeling methods with accuracy are essential to the performance prediction and optimal design of such channels. Up to now, there exists a variety of modeling methods including theoretical analytical method, equivalent circuit method, two-port network method, and finite element modeling method. However there is a little chaos about how to choose an appropriate modeling method among them. There is also a lack of a systematic modeling framework presented involving principles, advantages, disadvantages, and constraint conditions of these methods. In this review, a common modeling framework for through-metal-wall acoustic-electric channels based on piezoelectric transducers is presented. The principles of the major modeling methods are introduced and the adoptability and constraints of each method are discussed. For each modeling method, simulation and or experimental results are also presented to validate the accuracy of the corresponding modeling method.
\end{abstract}

\section{Introduction}

In general, wireless sensing and health monitoring of closed metal structures requires the use of physical penetrations and wire feedthrough to power embedded sensors. However, wire feedthrough takes disadvantages of limiting, or potentially compromising the structural integrity and environmental isolation. Traditional electromagnetic approaches for wireless transmission of power and signals are suppressed in these applications due to the strong Faraday shielding effect presented by the thick metal barriers, making them highly inefficient. In recent years, more and more attention has been paid to acoustic energy transmission by using ultrasonic wave through metal barrier as an alternative. The technology can be applied to the power transmission and data communication through sealed metallic containers.

Various systems or prototype systems have been developed. The majority of the acoustic-electric channels are designed for through planar metal barrier based on piezoelectric transducers (PZTs). Typically, a pair of piezoelectric transducers is coaxially bonded to opposite surfaces of a planar metal barrier of a hermetical metallic structure by an epoxy resin. The PZT outside of the metallic structure generates ultrasonic mechanical vibrations, which propagate through the planar metal barrier, and the internal transducer receives ultrasound waves and converts them into electric energy.

In order to evaluate and optimize the performance of the acoustic-electric channel, it is of great significance to model the acoustic-electric channel and analyze its characteristics including input impedance, output-to-input voltage ratio, and power transmission efficiency.

There have been a wide variety of methods for modeling and characterizing the channel such as analytical methods based on wave propagation equation and piezoelectric theory [1-7], equivalent circuit methods [8-17], finite element modeling (FEM) methods [10,11, 13, 18, 19], and the two-port network model based methods [20-22].

The above modeling methods can be applied to different applications. Some are useful only under ideal assumptions, some are suitable for the acoustic-electric channel through 
a planar metal barrier but not suitable for a curved metal barrier, and some are not applicable when channels are connected to external circuits (such as energy harvesting circuits, sensor monitoring circuits, modulation, and demodulation circuits). There is no modeling method that is suitable for all cases. In the process of designing the ultrasonic power transmission system, there is confusion about how to choose an appropriate modeling method from so many candidates. Till now, there is no modeling framework presented for acoustic-channels based on piezoelectric transducers. The advantages and disadvantages of each modeling method should be analyzed and compared in detail.

In this review, we first present principles of the main modeling methods and then discuss the applicable conditions and constraints of each method. In order to verify the validity of modeling methods, ultrasonic power transmission channels including through a planar metal wall and through a cylindrical metal pipe are established, respectively, to verify the effectiveness of various modeling methods. The simulation results are compared with the experimental results. There is no multipeak and multivalley characteristic curve, especially, for the established cylindrical pipe channel. This phenomenon is different from that of the planar metal barrier channel. At last, the modeling framework for throughmetal-wall acoustic-electric channels based on piezoelectric transducer has been established, which is expected to be helpful to optimal design and performance prediction of through-metal-wall ultrasonic power transmission channels.

\section{Theoretical Modeling Methods Based on Wave Propagation Equation and Piezoelectric Theory}

$\mathrm{Hu}$ et al. [1] first suggested the idea of using ultrasonic waves to transmit energy through planar metal wall based on piezoelectric material. The suggested acoustic-electric channel configuration is illustrated in Figure 1.

The planar metal plate represents the metal wall of a sealed armor sandwiched by a pair of piezoelectric layers, one for generating acoustic waves driven by an excitation voltage source and the other for converting the acoustic energy into electric energy to power the load $Z_{L}$.

The channel is driven into thickness-stretch vibrations by a harmonic voltage excitation. Just taking the thicknessstretch vibration mode into account, they have derived the mathematical model of the system based on the following acoustic wave equation and the linear piezoelectric equation:

$$
\begin{aligned}
c_{33} u_{3,33}+e_{33} \phi_{, 33} & =\rho \ddot{u}_{3} \\
e_{33} u_{3,33}-\varepsilon_{33} \phi_{, 33} & =0 \\
E_{3} & =-\phi_{, 3} \\
T_{33} & =c_{33} u_{3,33}-e_{33} E_{3} \\
D_{3} & =e_{33} u_{3,3}+\varepsilon_{33} E_{3}
\end{aligned}
$$

where $u_{3}, E_{3}$, and $D_{3}$ are the components of the mechanical displacement vector, the electric field vector, and the electric displacement vector along thickness direction $x_{3}$, respectively. $T_{33}$ is the components of the stress tensor; and $\phi$ denotes the electric potential; $c_{33}, e_{33}$, and $\varepsilon_{33}$ are the elastic, piezoelectric, and dielectric constants along $x_{3} . \rho$ is the mass density.

Combining with boundary conditions at the transmitting, receiving piezoelectric layers and the interface condition at the metal plate, they solved (1) and obtained analytical expressions for input impedance, output-to-input voltage ratio, and the power transmission efficiency of the channel. The numerical simulation results shown in Figure 2 illustrate that the output voltage of the system is magnified at the first several resonant frequencies of the system and there are peaks occurring in the power transmission efficiency curve versus driving frequency due to standing waves formed in the metal wall. The results indicate that the system may be used to charge the electronics inside the armor effectively at frequencies near the resonant frequency of the system.

But there are only numerical simulation results and no experimental channel established. Furthermore, some ideal assumptions during the theoretical analysis would not maintain the same as the actual application situations. For example the coupling layer is not taken into account during the analysis.

Later some other articles published by the same research group mainly focused on energy transmission through a cylindrical metal shell $[2,3]$, energy trapping characteristics $[4,5]$, connecting with energy harvesting circuit [6], and nonlinear behavior during the ultrasonic power transmission process [7]. All of them are based on the wave propagation equation and piezoelectric theory. But only numerical simulation results have been presented with extremely complex mathematical derivations.

The main disadvantages of the above theoretical modeling method lies in that the model cannot incorporate the head, tail mass, and the coupling layer and account for loss in all the mechanisms. In addition there is little mechanism presented for connecting the model to actual power processing circuitry (diodes, capacitors, rectifiers, etc.). Based on the acoustic-electric equivalent principles, equivalent circuit modeling methods are potential to overcome these shortcomings.

\section{Modeling Methods Based on Acoustic- Electric Equivalent Circuits}

Up to now, there are two main kinds of acoustic-electric equivalent circuits for modeling of through-metal-wall ultrasonic power transmission channels based on piezoelectric transducers. One is called Mason's equivalent circuit [8-15] and the other is Leach's equivalent circuit [15-17].

In order to illustrate the key points of the equivalent circuit based modeling methods, we take the example of modeling the wireless ultrasonic power transmission channel through a planar metal wall as shown in Figure 3. The channel is a sandwiched plate configuration, where two piezoelectric discs are stuck coaxially to the opposite side of the metal barrier with parallel faces by coupling layer of epoxy. A high frequency alternating power source ( $5 \mathrm{~V}$ peak sinusoidal) is applied to the electrodes of the transmitting transducer, 


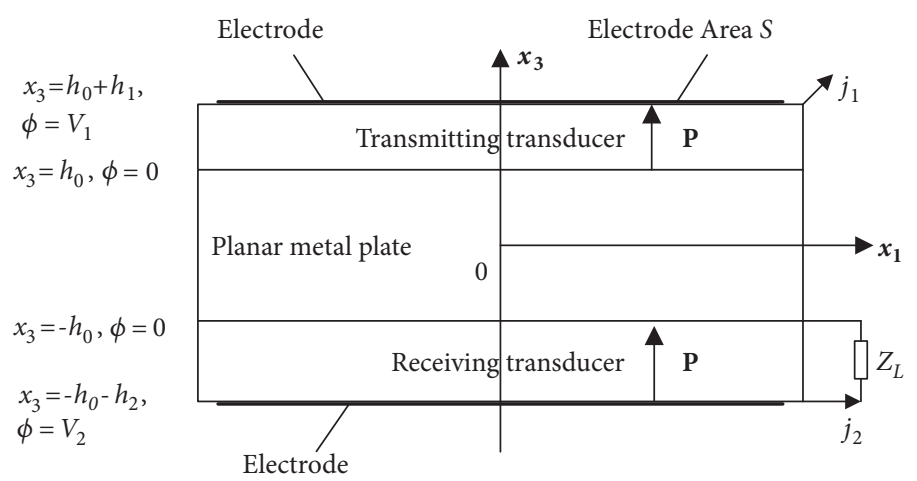

FIGURE 1: The acoustic-electric channel configuration formed with a planar metal plate sandwiched by two piezoelectric transducers, redrawn from $\mathrm{Hu}$ et al. [1].

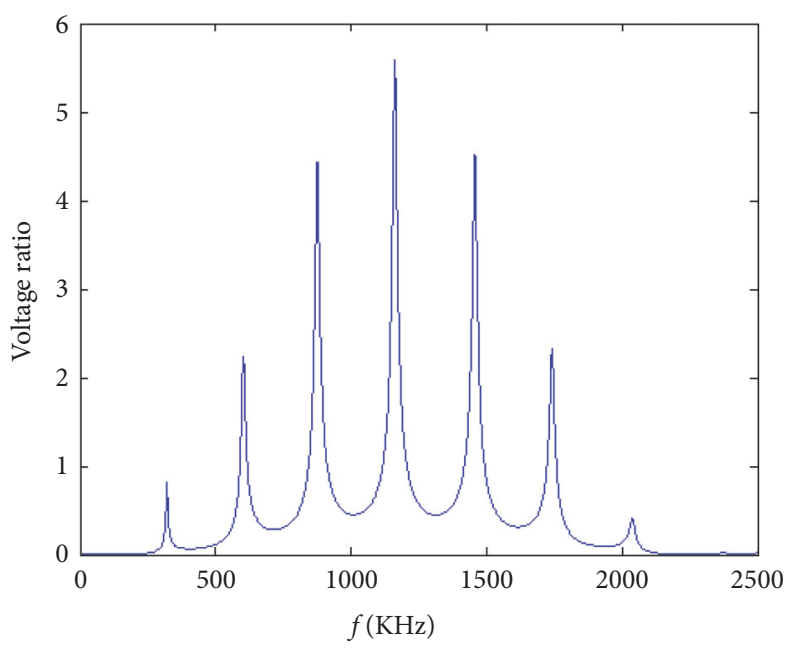

(a)

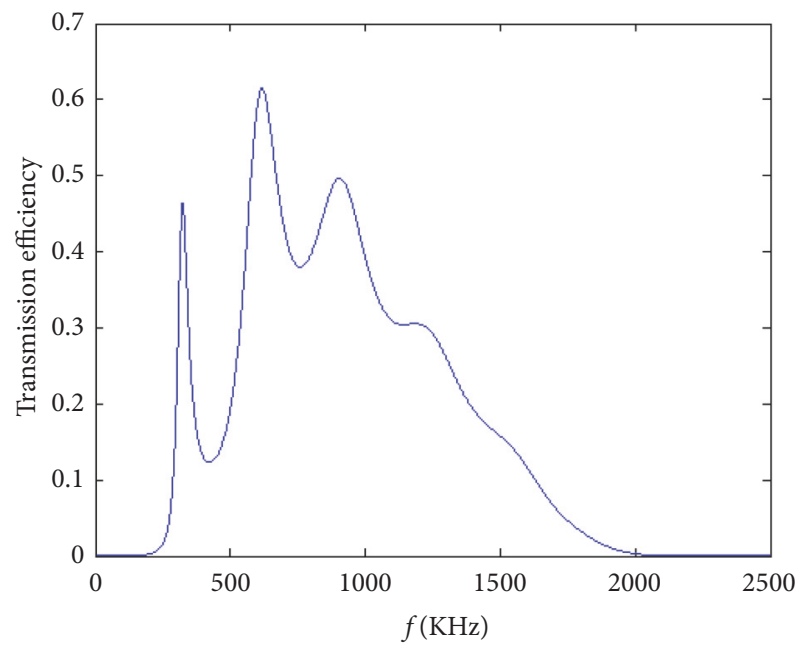

(b)

Figure 2: The output-input voltage ratio (a), the power transmission efficiency (b) versus the driving frequency, recalculation according to Hu et al. [1].

and a load resistor $(R=50 \Omega)$ is connected across the output electrodes of the receiving transducer on the receiving end.

3.1. Mason's Equivalent Circuit Modeling Method. Mason's circuit model has been introduced by Sherrit et al. [8]. They considered the effects of the back lining (air) for the same channel as $\mathrm{Hu}$ et al. [1]. The power transmission efficiency's other characteristics of the channel were obtained. In [15], Rao gave a detailed description of Mason's circuit modeling method.

To construct Mason's equivalent circuit model for the acoustic-electric channel, the key points are to establish Mason's equivalent circuit model for the piezoelectric transducer and for the nonpiezoelectric media layer such as the metal wall, epoxy coupling layers.

The nonpiezoelectric media layer is formed with a finite thickness $l$ in the $z$ direction of wave propagation, as illustrated in Figure 4. The left and right boundaries of the planar media layer/slab are denoted with $z_{1}$ and $z_{2}$, respectively. Both force $F_{1}$ and particle velocity $\dot{u}_{1}$ on the left slab face at $z_{1}$ are defined in the same positive $z$ direction as an incident

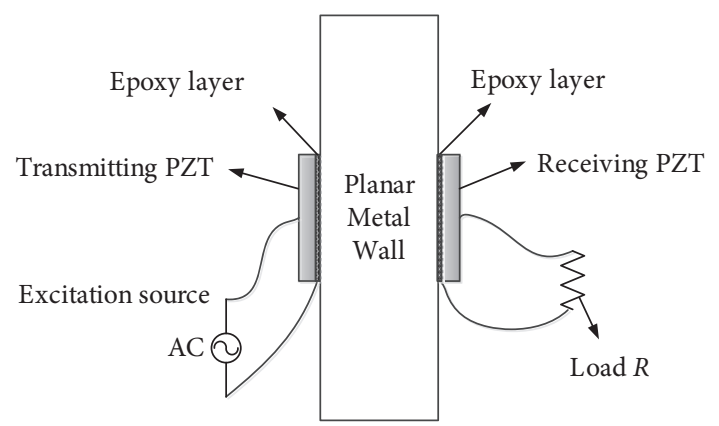

FIgURE 3: The wireless ultrasonic power transmission channel formed by a pair of PZTs through a planar metal wall.

wave entering the slab from the left. Similarly, both force $F_{2}$ and particle velocity $\dot{u}_{2}$ on the right slab face at $z_{2}$ are in the same positive $z$ direction as a transmitted wave leaving the slab to the right. Internally, the slab may contain both forward and backward traveling waves due to interfacial reflections from the acoustic media adjacent to the slab. 


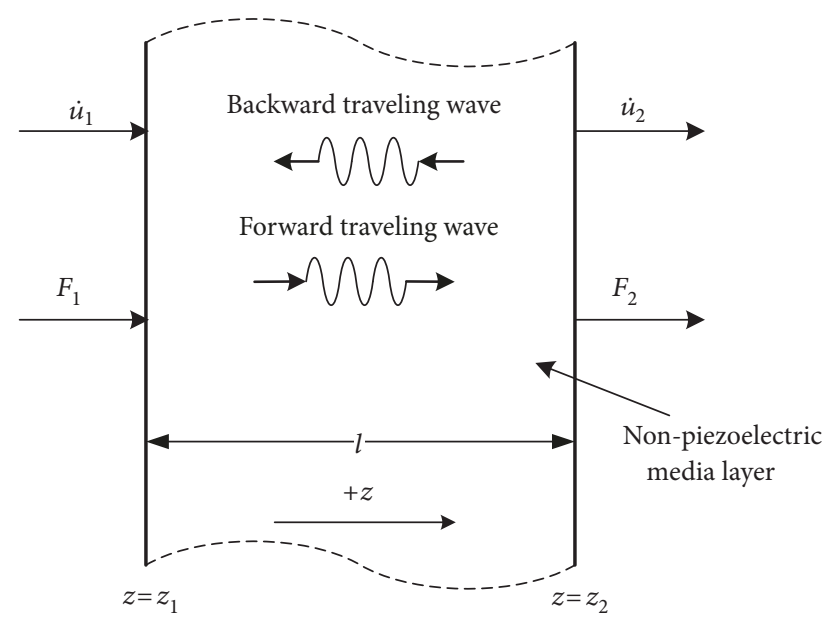

Figure 4: Force and particle velocity at the boundary and the pattern of waves inside of the nonpiezoelectric media layer excited by longitudinal wave.

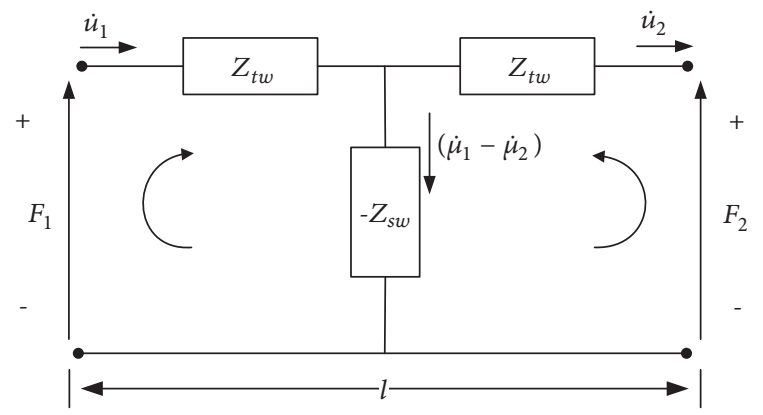

FIGURE 5: Mason's equivalent circuit of an acoustic nonpiezoelectric media layer of thickness $l$.

First, according to wave propagation equation and under the assumption of only thickness vibration mode, we can get the following set of equations [15]:

$$
\begin{aligned}
& F_{1}=\frac{Z_{e}}{i \sin \beta l}\left(\dot{u}_{1}-\dot{u}_{2}\right)+i Z_{e} \tan \frac{\beta l}{2} \dot{u}_{1} \\
& F_{2}=\frac{Z_{e}}{i \sin \beta l}\left(\dot{u}_{1}-\dot{u}_{2}\right)-i Z_{e} \tan \frac{\beta l}{2} \dot{u}_{2}
\end{aligned}
$$

where $\beta$ is known as propagation constant, defined as

$$
\beta=\omega \sqrt{\frac{\rho}{c}}=\frac{\omega}{v}
$$

where $\omega$ is the angular frequency and the variables $\rho$, $c$, and $v$ are the mass density, stiffness, and speed of sound in the material of the acoustic layer, respectively.

From physical acoustics, the acoustic impedance $Z_{e}$ of a medium with cross sectional area of $A$, is defined as

$$
Z_{e}=\sqrt{\rho c} A=\rho v A=\frac{c \beta}{\omega} A
$$

Now consider the electrical network in Figure 5.

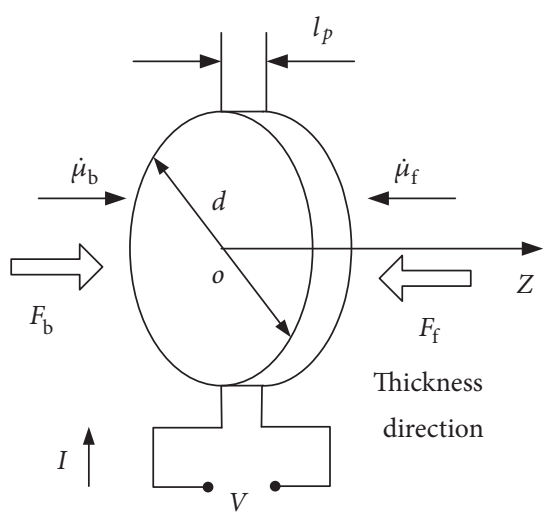

FIGURE 6: Diagrams of a piezoelectric disc transducer with thickness mode vibration and corresponding electrical interfaces.

According to Kirchoff's circuit theory, we can obtain the following equations for the electrical network:

$$
\begin{aligned}
& F_{1}=-Z_{s w}\left(\dot{u}_{1}-\dot{u}_{2}\right)+Z_{t w} \dot{u}_{1} \\
& F_{2}=-Z_{s w}\left(\dot{u}_{1}-\dot{u}_{2}\right)-Z_{t w} \dot{u}_{2}
\end{aligned}
$$

The impedance of the elements $Z_{s w}$ and $Z_{t w}$ are defined as

$$
\begin{aligned}
& Z_{s w}=i \frac{Z_{e}}{\sin \beta l} \\
& Z_{t w}=i Z_{e} \tan \frac{\beta l}{2}
\end{aligned}
$$

Substituting (6) to (5), we will get the same form of equation as (2). So the circuit shown in Figure 5 is just the equivalent circuit model for an acoustic nonpiezoelectric media layer. The velocity variables $\dot{u}_{1}, \dot{u}_{2}$ at the boundary surfaces of the media layer are equivalent to currents, and forces $F_{1}, F_{2}$ are equivalent to voltage. So it is an acousticelectric equivalent model.

For a piezoelectric transducer, it can be deemed as a piezoelectric crystal disc with diameter of $d$ and thickness of $l_{\mathrm{p}}$ shown as Figure $6 . F_{\mathrm{b}}, F_{\mathrm{f}}$ denote the forces experienced on the back and front faces of the PZT, respectively. $\dot{u}_{\mathrm{b}}, \dot{u}_{\mathrm{f}}$ are the velocity on surfaces, while $V$ is the voltage across two surfaces and $I$ is the current flowing across the PZT [15].

The constitutive piezoelectricity equations to describe the PZT can be expressed as

$$
\begin{aligned}
& T_{3}=c_{33}^{\mathrm{D}} S_{3}-h_{33} D_{3} \\
& E_{3}=-h_{33} S_{3}+\beta_{33}^{\mathrm{S}} D_{3}
\end{aligned}
$$

where $T_{3}, S_{3}$ are the stress and strain along $z$ direction, respectively. $c_{33}^{\mathrm{D}}$ is the complex elastic stiffness of the piezoelectric material measured with terminals of the PZT open. $D_{3}$ is the electric displacement along $z$ axis and $E_{3}$ is the electric field intensity. $\varepsilon_{33}^{S}$ is the clamped complex permittivity 
of the piezoelectric material along $z$ axis. And $h_{33}$ is the piezoelectric transmitting constant which is defined as

$$
h_{33}=\frac{e_{33}}{\varepsilon_{33}^{\mathrm{S}}}
$$

where $e_{33}$ is the piezoelectric stress constant along $z$ axis.

According to Newton's second law and after some mathematical derivations we can obtain the relation equations between $F_{\mathrm{b}}, F_{\mathrm{f}}$ and $\dot{u}_{\mathrm{b}}, \dot{u}_{\mathrm{f}}$ as

$$
\begin{aligned}
& F_{\mathrm{f}}=-i\left(\frac{Z a_{\mathrm{p}} A}{\tan \beta_{\mathrm{p}} l_{\mathrm{p}}} \dot{u}_{\mathrm{f}}+\frac{Z a_{\mathrm{p}} A}{\sin \beta_{\mathrm{p}} l_{\mathrm{p}}} \dot{u}_{b}+\frac{h_{33}}{i \omega} I\right) \\
& F_{\mathrm{b}}=-i\left(\frac{Z a_{\mathrm{p}} A}{\sin \beta_{\mathrm{p}} l_{\mathrm{p}}} \dot{u}_{\mathrm{f}}+\frac{Z a_{\mathrm{p}} A}{\tan \beta_{\mathrm{p}} l_{\mathrm{p}}} \dot{u}_{\mathrm{b}}+\frac{h_{33}}{i \omega} I\right)
\end{aligned}
$$

where $A$ is the area of the PZT disk. $\beta_{\mathrm{p}}$ is the propagation constant in the piezoelectric material. $\omega$ is the angular frequency of $I$. $Z a_{\mathrm{p}}$ is the acoustic impedance of the piezoelectric material which is defined as

$$
Z a_{\mathrm{p}}=\sqrt{\rho c_{33}^{\mathrm{D}}}=\rho v
$$

The voltage $V$ across the electrical terminals of the PZT is obtained by integrating the electric field intensity $E_{3}$ along the thickness of the PZT

$$
\mathrm{V}=\int_{0}^{l_{p}} E_{3} d z
$$

Finally, we can get the expression of voltage $V$ as

$$
V=-i\left(\frac{h_{33}}{\omega} \dot{u}_{\mathrm{f}}+\frac{h_{33}}{\omega} \dot{u}_{\mathrm{b}}+\frac{1}{\omega C_{0}} I\right)
$$

where $C_{0}$ is the clamped capacitance of PZT defined as

$$
C_{0}=\frac{\varepsilon_{33}^{S} A}{l_{\mathrm{p}}}
$$

We can cast (9), (12) into a matrix form as follows:

$$
\left[\begin{array}{c}
F_{\mathrm{f}} \\
F_{\mathrm{b}} \\
V
\end{array}\right]=-i\left[\begin{array}{ccc}
\frac{Z a_{\mathrm{p}} A}{\tan \beta_{\mathrm{p}} l_{\mathrm{p}}} & \frac{Z a_{\mathrm{p}} A}{\sin \beta_{\mathrm{p}} l_{\mathrm{p}}} & \frac{h_{33}}{\omega} \\
\frac{Z a_{\mathrm{p}} A}{\sin \beta_{\mathrm{p}} l_{\mathrm{p}}} & \frac{Z a_{\mathrm{p}} A}{\tan \beta_{\mathrm{p}} l_{\mathrm{p}}} & \frac{h_{33}}{\omega} \\
\frac{h_{33}}{\omega} & \frac{h_{33}}{\omega} & \frac{1}{\omega C_{0}}
\end{array}\right]\left[\begin{array}{c}
\dot{u}_{\mathrm{f}} \\
\dot{u}_{\mathrm{b}} \\
I
\end{array}\right]
$$

Based on (14), the equivalent circuit model can be constructed as shown in Figure 7.

Figure 7 is called Mason's equivalent circuit model for a PZT, in which $Z_{t}, Z_{s}$ denote the equivalent electric impedances. A transformer with the number of turn's ratio $N$, a capacitor with value of $C_{0}$, and a negative capacitor with value of $-C_{0}$ are also included in the model. $C_{0}$ is just the value

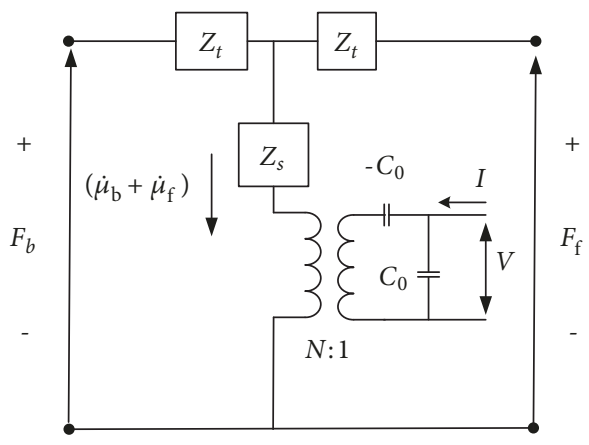

FIGURE 7: Mason's equivalent circuit model for a piezoelectric disc transducer.

of the clamped capacitance of the PZT; the other quantities are defined as

$$
\begin{aligned}
& Z_{s}=Z a_{\mathrm{p}} A \csc \beta_{\mathrm{p}} l_{\mathrm{p}} \\
& Z_{t}=Z a_{\mathrm{p}} \tan \left(\frac{\beta_{\mathrm{p}} l_{\mathrm{p}}}{2}\right) \\
& N=C_{0} h_{33}
\end{aligned}
$$

Based on Mason's equivalent circuit models for a single piezoelectric transducer and the nonpiezoelectric media layer presented previously, we can make a cascade of Mason's equivalent circuit models for transmitting PZT, transmitting coupling layer, planar metal wall, receiving coupling layer, and receiving PZT. Thus Mason's equivalent circuit model for the acoustic-electric channel through a planar metal wall based on a pair PZTs can be obtained as illustrated in Figure 8. $Z_{t w 1}$ and $Z_{s w 1}$ denote the equivalent impedances related to the planar metal wall; $Z_{t w 2}$ and $Z_{s w 2}$ denote the equivalent impedances related to the coupling layers; since assuming that transmitting side coupling layer is the same as that of the receiving side, both have the same impedances. $Z_{k}$ denotes the impedance of the back layer of the transmitting and receiving PZT, Here the back layer is air, so there is the same impedance.

Since the whole model of the channel has been established, we can use this model to obtain the characteristics of the channel such as input impedance, output-input voltage ratio, and power transmission efficiency of the channel and. We may develop calculation program using MATLAB to perform the circuit calculations to get such characteristics.

Can we use circuit simulation software like SPICE to analyze the model? Unfortunately, we cannot do this. In Mason's equivalent circuit model, there are a negative capacitor, an ideal transformer and impedance elements which are defined in terms of transcendental functions. Circuit simulation software cannot treat such elements. Furthermore, it is not easy to analyze the characteristics if more complex external circuits are added to the channel while Leach's equivalent circuit model only uses standard lumped components to represent the PZT and other nonpiezoelectric acoustic layers, which will facilitate the use of software tools that are already available for circuit analysis and simulation. 


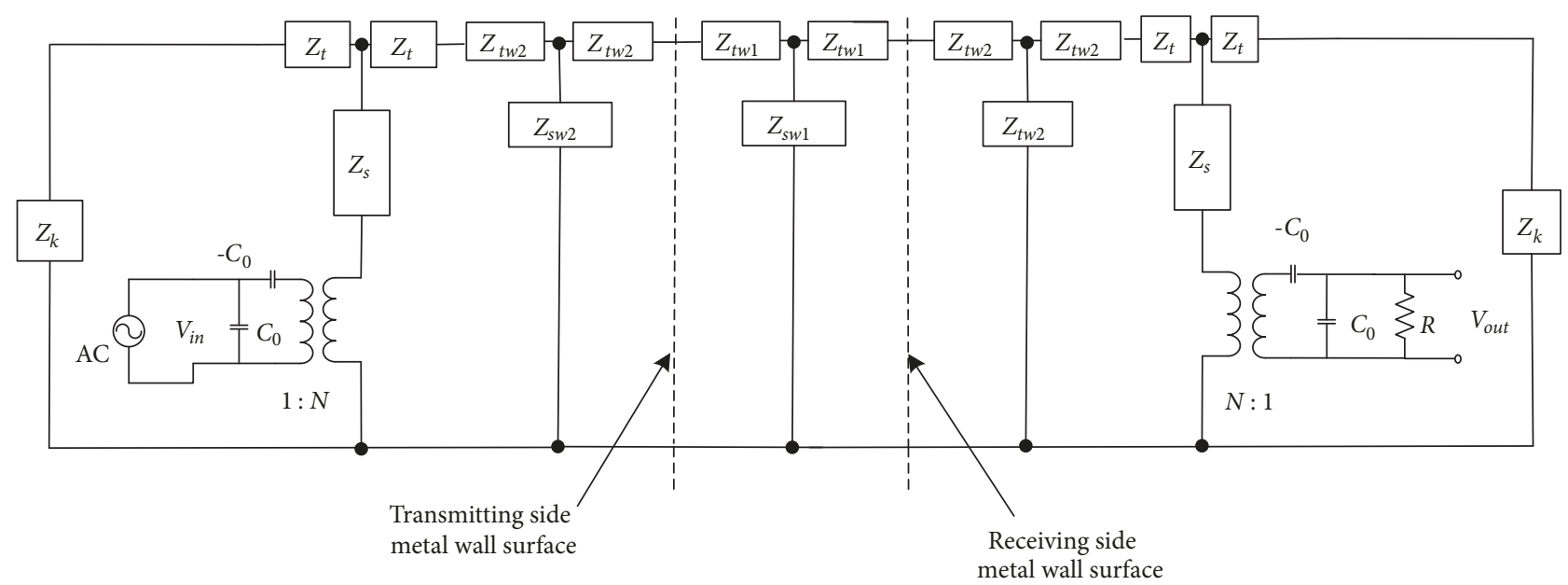

FIGURE 8: Mason's equivalent circuit model for the acoustic-electric channel based on a pair of PZTs.

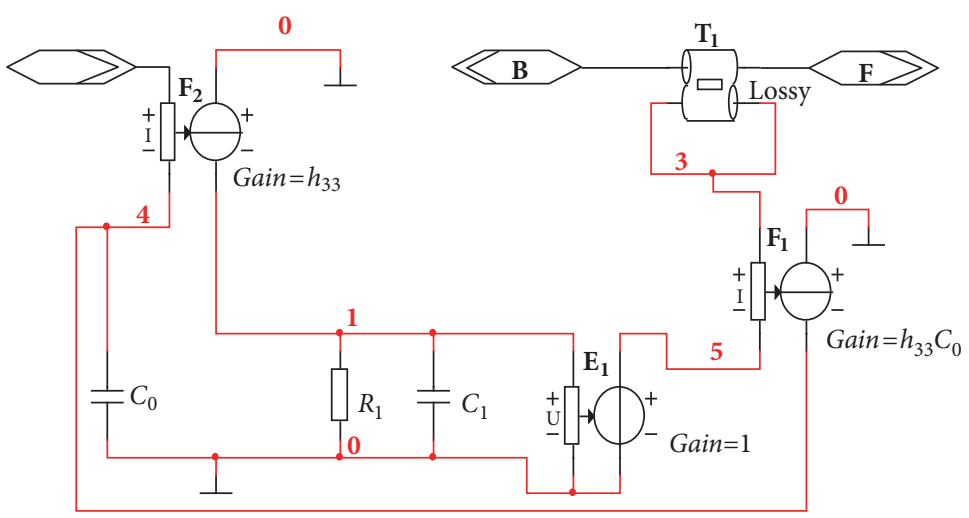

FIGURE 9: Leach's equivalent circuit model for a piezoelectric transducer [23].

3.2. Leach's Equivalent Circuit Modeling Method. The main distinctions between Leach's equivalent circuit model and Mason's equivalent circuit model lie in the ways of modeling the piezoelectric transducer and nonpiezoelectric media layers.

Leach's equivalent circuit model for a PZT is presented in Figure 9 which can be simulated in SPICE circuit simulation software [17]. Leach's equivalent circuit model for a piezoelectric transducer has three ports. Two mechanical ports denoted by $\mathbf{B}$ and $\mathbf{F}$ represent the back and front of a piezoelectric transducer, and a single electrical port $\mathbf{E}$ is assigned to the assumption that the opposite side of the piezoelectric transducer is grounded. The internal electric symbols in the model denoted by $\mathbf{F}_{1}, \mathbf{F}_{2}$ represent two voltagecontrolled voltage sources and $\mathbf{E}_{1}$ is a current-controlled current source [23].

The parameter $h_{33}$ has the same definition as (8), and the clamped capacitance $C_{0}$ is defined the same as (13). $\mathbf{T}_{\mathbf{1}}$ represents the lossy transmission line of the piezoelectric transducer using four parameters $L, C, R$, and $G$ to describe, which are defined as

$$
\begin{aligned}
& L=A \rho \\
& C=\frac{1}{A \rho c^{2}} \\
& R=\frac{\omega L}{Q_{m}}
\end{aligned}
$$

where $\omega$ is excitation angular frequency, and the variables $A, \rho, Q_{m}$, and $c$ are the PZT disc area, the mass density, the mechanical quality factor, and speed of sound in the piezoelectric crystal, respectively. Because the loss due to thermal conduction is negligible, usually the conductance $G$ takes the value of zero.

It should be pointed out that the combination of $R_{1}$, $C_{1}, \mathbf{F}_{1}, \mathbf{F}_{2}$ and $\mathbf{E}_{1}$ replaces the functions of the negative capacitance and the ideal transformer in Mason's circuit model. According to literature [15-17], usually $R_{1}$ takes value of $1 \mathrm{k} \Omega$ and $C_{1}$ takes $1 \mathrm{~F}$.

Leach's model also eliminates the impedance elements $Z_{s}$ and $Z_{t}$ in Mason's equivalent circuit model, which have 


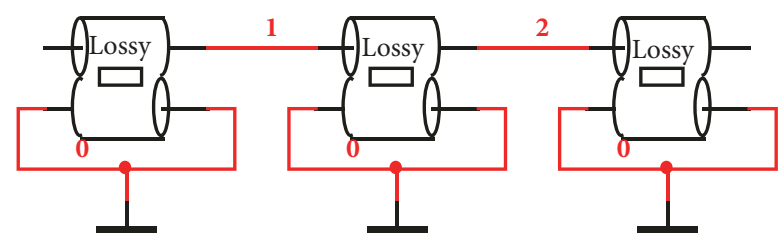

FIGURE 10: Equivalent lossy transmission line model of three cascaded dissipative acoustic layers [23].

been replaced by an electrical lossy transmission line $\mathbf{T}_{\mathbf{1}}$. The advantage of this modification is that the transmission line $\mathbf{T}_{1}$ representing the propagation of acoustic waves across the PZT interfaces very well with transmission lines that represent other acoustic layers such as coupling epoxy layers.

Because the propagation of mechanical waves in acoustic layers is analogous to the propagation of electrical waves in lossy transmission lines, we can also use lossy transmission lines to represent the metal barrier and the coupling layers. Supposing that the PZT are coupled to the metal wall using epoxy, there are epoxy-wall-epoxy acoustic layers between the transmitting and receiving PZTs. These cascaded acoustic layers can be represented as a set of lossy transmission lines connected in series, where one lossy transmission line represents each acoustic layer. The equivalent lossy transmission line model of the three cascaded acoustic layers is shown in Figure 10.

The calculation of the lossy transmission line parameters for the metal barrier is the same as that of the piezoelectric transducer. For epoxy layer, the calculation of the corresponding lossy transmission line parameters $L, C$, and $G$ is the same as that of the piezoelectric transducer. But it is different for $R$; $R$ is calculated according to the equation of $R=2 \rho c A \alpha$, where $\alpha$ is the coefficient of attenuation due to viscous losses of the epoxy layer.

In this way the whole Leach's equivalent circuit model for the wireless ultrasonic power transmission channel is established as shown in Figure 11.

We can simulate and analyze the characteristics of the channel model in SPICE software environment. For verifying the models, an experimental ultrasonic channel is constructed and measurements of the input impedance are carried out. In experiment, the material of the transmitting and receiving piezoelectric transducers is PIC155. The transducer's resonance frequency is $2.009 \mathrm{MHz}$. The material properties and geometric parameters of the piezoelectric transducer are listed in Table 1, and material properties of other parts are listed in Table 2.

Figure 12 shows the constructed channel's input impedance modulus and phase curves versus frequency from Mason's equivalent circuit model, Leach's equivalent circuit model, and experimental measurements. It can be observed that the impedance curve from Mason's circuit is well matched to that from Leach's circuit throughout the frequency range except for some small deviations. There are multiple peaks and valleys appearing in impedance curves with a certain frequency interval which are due to the standing waves formed in the planar metal barrier. The resonance frequency of the acoustic-electric channel is located nearby $2.3 \mathrm{MHz}$, which is a bit deviation from that of the piezoelectric transducer.

Although there are some deviations between the experimental measurement results and the model simulation results, the trends of the impedance curves versus frequency are in the same way. There are also peaks and valleys in the measurement results. The deviations may be due to the parameters measurement errors of the PZT and acoustic material, imperfect coupling between epoxy and the metal barrier, the noise interference, etc.

Beside the two above mentioned equivalent circuit models, another equivalent circuit model for piezoelectric transducer is the Butterorth-Van Dyke model, commonly known as the BVD model [15]. In this model, the PZT is represented by a clamped capacitance in parallel with a RLC series circuit. However this model can only describe the behavior of the PZT near its fundamental frequency resonance, and we will not discuss it in detail here.

As a summary, equivalent circuit based modeling methods are superior to theoretical modeling methods. The equivalent circuit model can be easily modified to take additional acoustic media such as coupling layers and insulation layers into account. Other electric circuits including power harvesting circuit and sensor conditioning circuit can be connected directly to the model while the theoretical modeling method will need a great deal of complex and difficult calculations which make it impractical to use for the study of ultrasonic systems with a larger number of components. Leach's equivalent circuit model and Mason's equivalent circuit model can obtain the accordant characteristics for the same channel. Furthermore, Leach's equivalent circuit modeling method gains advantage over Mason's equivalent circuit modeling method because the former can simulate and analyze the ultrasonic channel with circuit simulation software, while the latter contains components such as the negative capacitor and the ideal transformer and cannot be used directly in circuit simulation software. Experimental results have indicated that equivalent circuit modeling methods are applicable to the cases where the metal barrier is planar with certain accuracy.

As we mentioned, there are a variety of factors influencing characteristics of the acoustic-electric power transmission system, such as the environment temperature, the coupling quality, and even the geometric shape of the metal barrier. Under these circumstances, usually it is difficult to build up an accurate equivalent circuit model due to the complexity and uncertainty of channel parameters, while finite element modeling method can fulfill the modeling task in such occasions.

\section{Finite Element Modeling Method}

Finite element modeling method which belongs to a kind of theoretical analysis method has been used to model and analyze the output to input voltage ratio and power transmission efficiency of ultrasonic power transmission channels through planar metallic barriers with professional FEM software like ANSYS [10, 11, 13] or COMSOL Mutiphysics [18, 19]. The simulation results from the finite element model are similar 


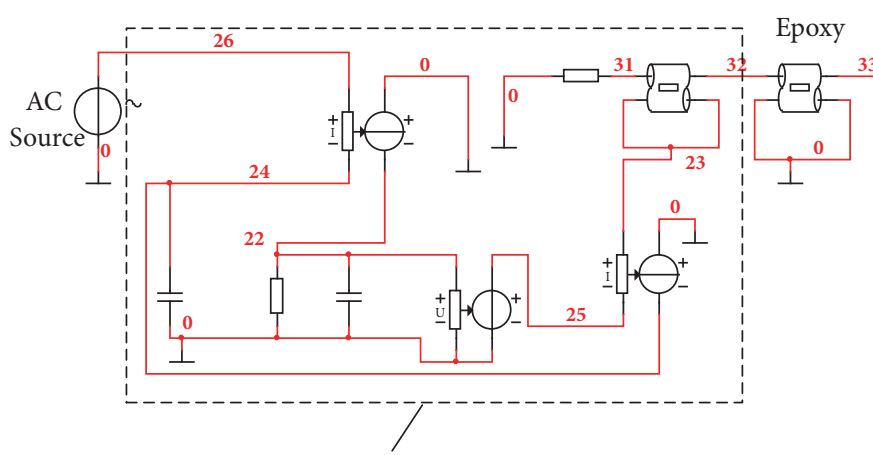

Transmitting transducer model

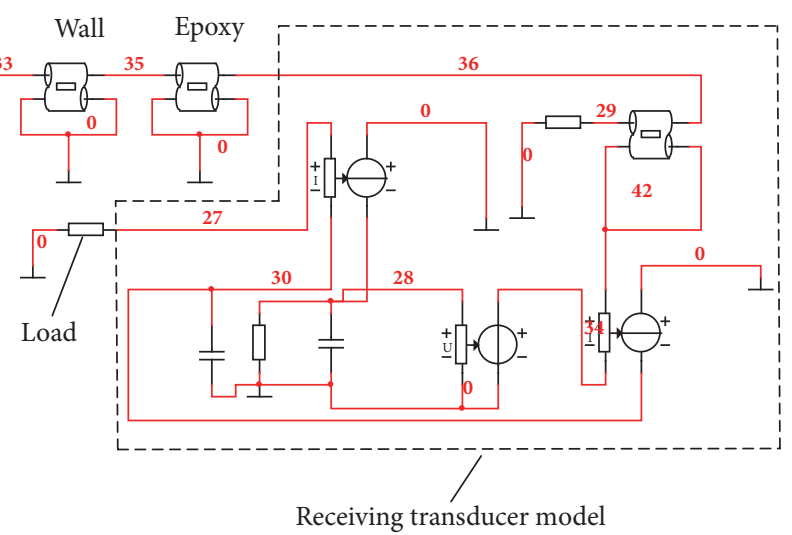

FIGURE 11: Leach's equivalent circuit model of the ultrasonic wireless power transmission channel through a planar metal wall for SPICE simulation [23].

TABLE 1: Material properties and geometric dimensions of PIC155 piezoelectric transducer [23].

\begin{tabular}{ccccccccc}
\hline Transducer & $d(\mathrm{~mm})$ & $l_{p}(\mathrm{~mm})$ & $\rho\left(\mathrm{kg} / \mathrm{m}^{3}\right)$ & $h_{33}(\mathrm{~V} / \mathrm{m})$ & $\varepsilon_{33}^{S}(\mathrm{~F} / \mathrm{m})$ & $e_{33}\left(\mathrm{C} / \mathrm{m}^{2}\right)$ & $Q_{m}$ & $c(\mathrm{~m} / \mathrm{s})$ \\
\hline PIC155 & 20 & 1 & 7800 & $1.95 \times 10^{9}$ & $6.726 \times 10^{-9}$ & 13.12 & 80 & 3772 \\
\hline
\end{tabular}
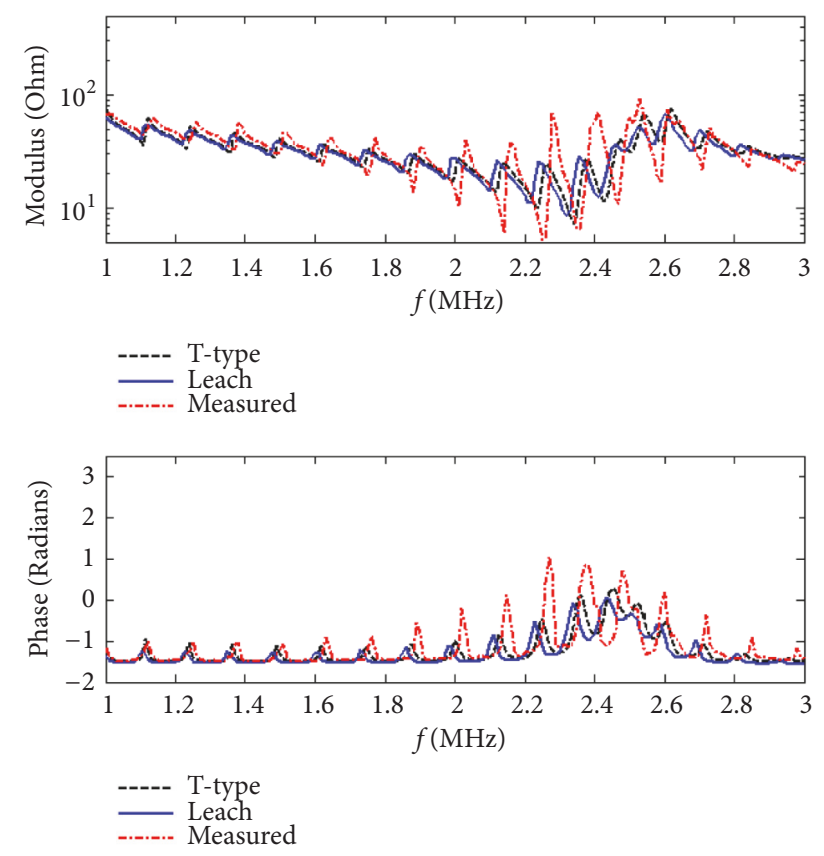

FIGURE 12: The input impedance of the constructed ultrasonic channel through a planar metal barrier [23].

TABLE 2: Material properties and geometric dimensions of other parts of the channel [23].

\begin{tabular}{lccc}
\hline Material & Thickness $(\mathrm{mm})$ & $\rho\left(\mathrm{kg} / \mathrm{m}^{3}\right)$ & $c(\mathrm{~m} / \mathrm{s})$ \\
\hline Stainless Steel & 22 & 7850 & 5790 \\
Epoxy & $32 \times 10^{-3}$ & 1160 & 2620 \\
\hline
\end{tabular}

to that from the equivalent circuit modeling method for the case of through a planar metallic wall.
But when the metal barrier has a curved surface, it is not easy to model the acoustic-electric channel using equivalent circuit. The main difficulties lie in that the curved metal barrier and coupling layers cannot be represented by using the lossy transmission lines like a planar barrier. For modeling channels through metal barrier with complex geometric shape, FEM modeling method has its peculiar advantages, which can be applicable to metal barriers with an arbitrary geometric shape. Here we use the three-dimensional COMSOL finite element method for modeling and analyzing the ultrasonic power transmission channel through a cylindrical metal pipe.

In our study, a pair of piezoelectric transducers with diameter of $10 \mathrm{~mm}$ and thickness of $1 \mathrm{~mm}$ is aligned concentrically on the inside and outside of a cylindrical metal pipe by epoxy. Because the PZT is a planar disk, it is necessary to add a thin curved transition piece between the transducer and the surface of the cylindrical metal barrier, and the thinnest thickness of the transition piece is $2 \mathrm{~mm}$; the diameter of the transition piece is the same as the transducer. The material of the pipe and the transition piece is stainless steel. The inner diameter of the metal pipe is $75 \mathrm{~mm}$, the external diameter is $85 \mathrm{~mm}$, and length is $200 \mathrm{~mm}$ [23].

The 3-dimensional geometric model of the channel formed by the cylindrical metal pipe, transition pieces, and PZTs is shown in Figure 13, which is established in SolidWorks software. The geometric model can be imported into COMSOL for performing further finite element calculation and analysis.

In simulation, an alternative source with voltage of $5 \mathrm{~V}$ is applied to Face 1 of the transmitting transducer as the electric excitation, and the opposite face of the transducer is grounded. A pure resistive load $(50 \Omega)$ is connected between Face 2 and the opposite face of the receiving transducer. In COMSOL, the stainless steel pipe, transition piece, and the epoxy are defined as the sound field model and the 


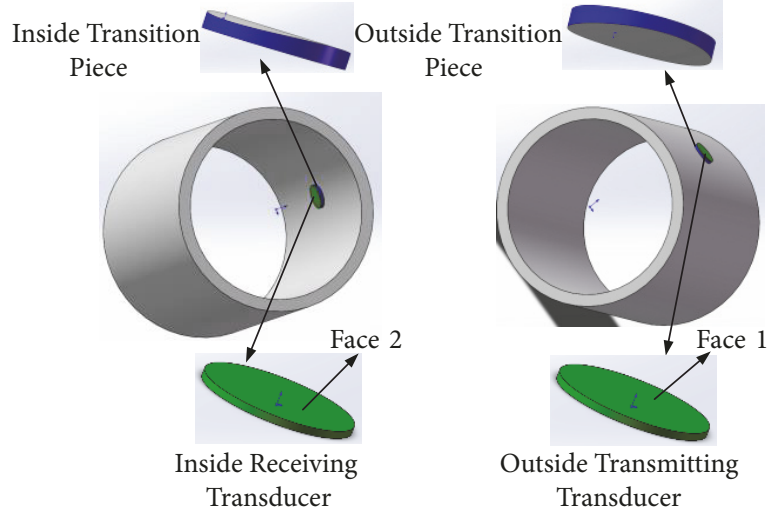

FIGURE 13: 3-dimensional geometric model of the ultrasonic power transmission channel through a cylindrical metal pipe [23].
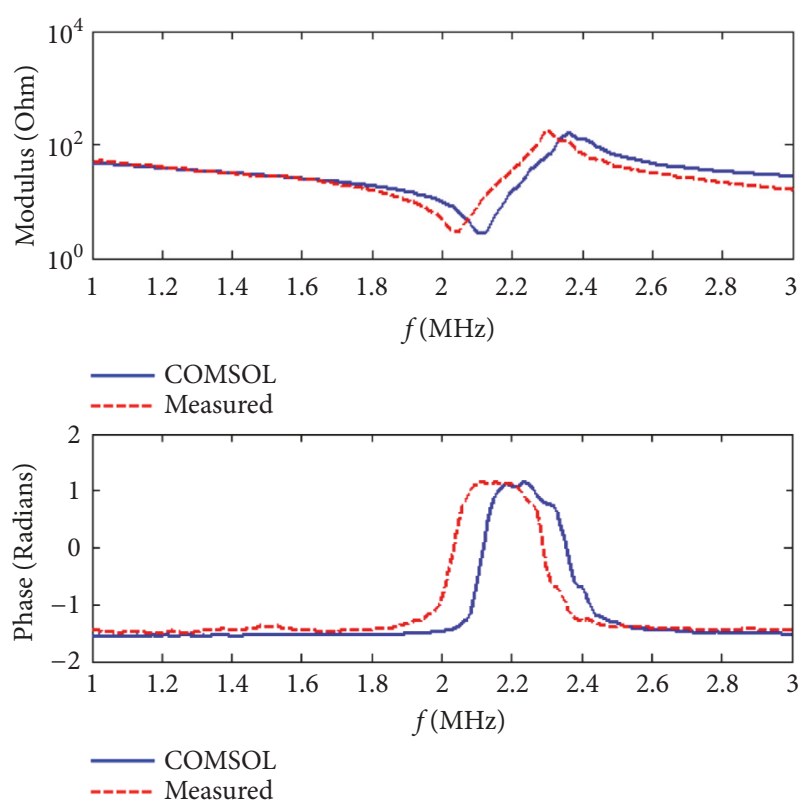

FIGURE 14: Input impedance characteristics of the channel through a cylindrical metal pipe [23].

contact surface of the epoxy and the transducer is defined as the sound solid coupling contact surface, and the other boundaries are defined as the hard sound field boundaries. Then the grid is divided and the frequency domain solution is performed. After a large amount of calculations, the outputto-input voltage ratio and equivalent input impedance of the channel are obtained. The physical channel is built up and experimental measurement is also carried out for comparison with the FEM simulation results.

Figure 14 shows the input impedance modulus and phase curves versus frequency from COMSOL simulation and experimental measurement. It can be observed that the impedance curve from finite element simulation is nearly matched to that from measurement except for a bit of frequency shift which may be caused by the geometric error of the actual channel, coupling effect, and so on. Another thing noticeable is that there are no multiple peaks and

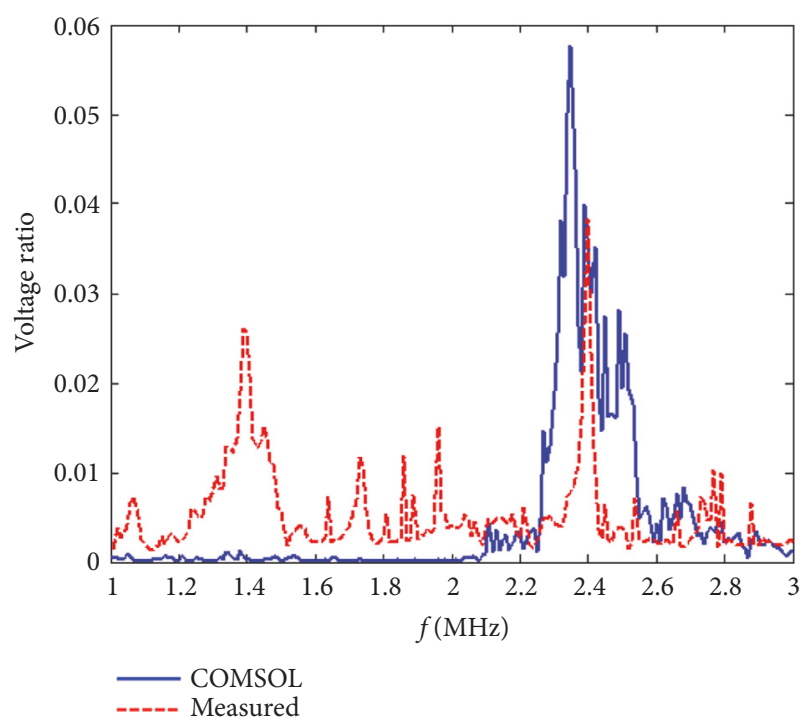

FIGURE 15: Output-to-input voltage ratio of the channel through a cylindrical metal pipe [23].

valleys in both simulation and experimental measurement results which is different from the phenomenon occurring in impedance curves of the channel through a planar metal barrier.

The output-input voltage ratio of the channel through the cylindrical metal barrier is shown in Figure 15. We can see that there are some deviations between the measured and simulated results in the frequency range of $1.2-1.5 \mathrm{MHz}$. The possible reason is that the actual configuration of the channel is different from that of simulation. As we know, the PZT transducers adhered to the inner and outer curve walls are not strictly symmetrical and the thickness of epoxy layer is not uniform, which may also lead to incomplete alignment of internal and external piezoelectric plates. While the accuracy of FEM results fully relies on the accurate configuration and parameters of all parts forming the channel, in order to make FEM model simulate the actual system more accurately, it is necessary to model the channel more carefully and precisely. There is a bit better correlation after $1.5 \mathrm{MHz}$. Both curves show the maximum voltage transfer frequency is near at $2.3 \mathrm{MHz}$. The voltage transfer ratio through the cylindrical metal wall is small and the measured maximum ratio is less than 0.05 , which means that it is more difficult for power delivery through a cylindrical metal wall than through a planar wall. Nevertheless, the 3-dimensional finite model is shown to have the potentiality in modeling the wireless ultrasonic power transmission system through a cylindrical metal barrier with certain accuracy.

Apart from the usual voltage transfer ratio, the input impedance characteristic, the temperature distribution and acoustic field distribution in the metal barrier can also be obtained using finite element modeling method. However the accuracy of simulation results fully relies on the accurate parameters of all parts forming the channel, and there is also a lack of understanding of the internal mechanism of the acoustic-electric channel. 


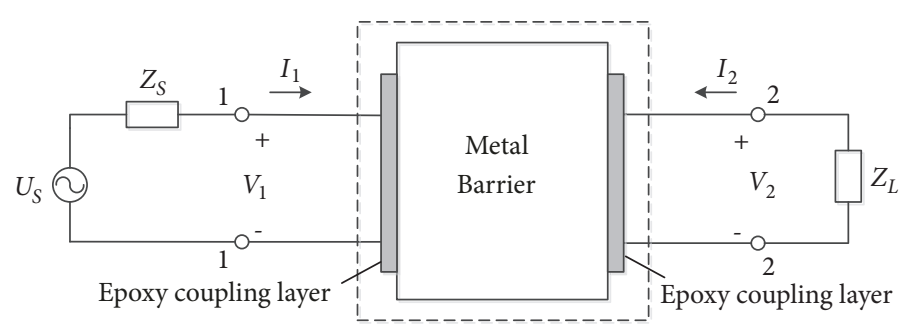

FIGURE 16: A two-port network model for through-metal-wall ultrasonic power transmission channel.

When the parameters of the material and parts are not clear or available, the aforementioned modeling methods are powerless. Especially if the acoustic-electric channel has already been established, we can analyze characteristics of the channel using two-port network modeling method.

\section{Modeling Method Based on Two-Port Network}

As for the wireless ultrasonic power transmission system configure shown in Figure 3, the channel consisting of a pair of piezoelectric transducers, epoxy coupling layers, and planar metal barrier can be viewed as a 'black box'. What is actually our concern are the input current and voltage at the input port and the current and voltage through the load. We do not care about the specific parameters of the parts forming the channel actually. Such a black box with inputoutput ports can be described using a two-port network as shown in Figure 16, where port 1 is driven by a power source $U_{s}$ with the internal impedance $Z_{s}$ and port 2 is connected with a load impedance $Z_{L}$.

Characteristics of the channel depended on the material, the shape, and size of the PZTs, the material, and thickness of the metal barrier, and the load impedance. Environment parameters such as temperature and humidity will also have impacts on the channel characteristics. For some actual engineering applications, the accurate parameters of the channel and environment conditions are difficult to obtain. So the accurate model of the channel is not easy to establish using aforementioned modeling methods. In such cases, we can first measure the network parameters of the two-port network without requirements of prior knowledge of the channel parts parameters. The channel characteristics such as the input impedance, the voltage transfer ratio, and the power transmission efficiency can then be obtained based on the measured two-port network parameters.

The network parameters are the intrinsic characteristics of a two-port network which are independent of the excitation source or the load impedance. A two-port network can be described using different types of parameters such as impedance ( $Z$-parameters), admittance ( $Y$-parameters), hybrid ( $h$-parameters), transmission ( $A B C D$ parameters), or scattering ( $S$-parameters) [24]. One type of parameters can then be converted into another type based on a set of mathematical formulas. Theoretically a two-port network can be described using any type of these parameters. However for a given network a certain type of parameters may not exist, and some types of parameters may be not easy to measure or obtain.

$Z$-parameters are the most common used network parameters. For the channel shown in Figure 16, denote the input voltage and current at port 1 as $V_{1}$ and $I_{1}$ and the output voltage and current at port 2 as $V_{2}$ and $I_{2}$; the relationship between these voltages and currents can be described using the following equation:

$$
\left[\begin{array}{l}
V_{1} \\
V_{2}
\end{array}\right]=\left[\begin{array}{ll}
Z_{11} & Z_{12} \\
Z_{21} & Z_{22}
\end{array}\right]\left[\begin{array}{l}
I_{1} \\
I_{2}
\end{array}\right]
$$

The impedance matrix $\mathbf{Z}=\left[\begin{array}{ll}Z_{11} & Z_{12} \\ Z_{21} & Z_{22}\end{array}\right]$ is referred to $Z$-parameters of the two-port network, where $Z_{11}=$ $\left.\left(V_{1} / I_{1}\right)\right|_{I_{2}=0}, Z_{12}=\left.\left(V_{1} / I_{2}\right)\right|_{I_{1}=0}, Z_{21}=\left.\left(V_{2} / I_{1}\right)\right|_{I_{2}=0}, Z_{22}=$ $\left.\left(V_{2} / I_{2}\right)\right|_{I_{1}=0} ; I_{1}=0$ represents that port 1 is in open circuit and $I_{2}=0$ represents port 2 is open.

$Z$-parameters can be calculated according to the definition by measuring the voltage and current at the input or at the output port with the other port remaining open. After obtaining $Z$-parameters, we can use $Z$-parameters to analyze the network's characteristics. As we know, $Z_{11}$ is just the input impedance of the channel.

$A B C D$ parameters, which are very useful in describing serial-cascaded two-port networks relate $V_{1}, I_{1}$ and $V_{2},-I_{2}$ as follows:

$$
\left[\begin{array}{l}
V_{1} \\
I_{1}
\end{array}\right]=\left[\begin{array}{ll}
A & B \\
C & D
\end{array}\right]\left[\begin{array}{c}
V_{2} \\
-I_{2}
\end{array}\right]
$$

To get two serial-cascaded two-port network's $A B C D$ parameters we just need to multiply each two-port network's $A B C D$ matrix [20]. $Z$-parameters can be converted into $A B C D$ parameters using the following formulas:

$$
\begin{aligned}
& A=\frac{Z_{11}}{Z_{21}}, \\
& B=\frac{Z_{11} Z_{22}-Z_{12} Z_{21}}{Z_{21}}, \\
& C=\frac{1}{Z_{21}}, \\
& D=\frac{Z_{22}}{Z_{21}}
\end{aligned}
$$

$S$-parameters use incident and reflected power waves at the input and output ports to describe the two-port 
network. Under radio frequency range (ten megahertz order of magnitude), it is difficult to directly measure $V_{1}, I_{1}$ and $V_{2}$, $I_{2}$ because simply opening the network's port will produce electromagnet power radiation. While $S$-parameters can overcome this shortage, for power waves can be conveniently measured using the power coupler [25], the acoustic-electric channel described using power waves is shown in Figure 17.

where $\widehat{a}_{G}, \widehat{b}_{G}$ represent the incident and reflected power waves of the power source $U_{s}$. $\widehat{a}_{1}, \widehat{b}_{1}$ represent the incident and reflected power waves at port 1 of the channel, and $\hat{a}_{2}$, $\widehat{b}_{2}$ represent the incident and reflected power waves at port 2 , respectively, while $\widehat{a}_{L}, \widehat{b}_{L}$ represent the incident and reflected power waves of the load $Z_{L}$. There exists the following equation relationship:

$$
\begin{aligned}
& \widehat{a}_{G}=\widehat{b}_{1}, \\
& \widehat{a}_{1}=\widehat{b}_{G}, \\
& \widehat{a}_{L}=\widehat{b}_{2}, \\
& \widehat{a}_{2}=\widehat{b}_{L}
\end{aligned}
$$

The relationship between incident and reflected power waves at the input and output ports of the channel can be described as

$$
\left[\begin{array}{l}
\hat{b}_{1} \\
\widehat{b}_{2}
\end{array}\right]=\left[\begin{array}{ll}
S_{11} & S_{12} \\
S_{21} & S_{22}
\end{array}\right]\left[\begin{array}{l}
\hat{a}_{1} \\
\widehat{a}_{2}
\end{array}\right]
$$

where $S_{11}, S_{12}, S_{21}$, and $S_{22}$ represent scattering parameters of the channel, and scattering parameters of a two-port network can also be conveniently measured by a network analyzer with a reference impedance $Z_{0}$ [22]. The following equations give the relationship between power waves and voltages and currents at port 1 and port 2 .

$$
\begin{aligned}
& \widehat{a}_{1}=\frac{1}{2}\left(\frac{V_{1}}{\sqrt{Z_{0}}}+I_{1} \sqrt{Z_{0}}\right), \\
& \widehat{b}_{1}=\frac{1}{2}\left(\frac{V_{1}}{\sqrt{Z_{0}}}-I_{1} \sqrt{Z_{0}}\right) \\
& \widehat{a}_{2}=\frac{1}{2}\left(\frac{V_{2}}{\sqrt{Z_{0}}}+I_{2} \sqrt{Z_{0}}\right), \\
& \widehat{b}_{2}=\frac{1}{2}\left(\frac{V_{2}}{\sqrt{Z_{0}}}-I_{2} \sqrt{Z_{0}}\right)
\end{aligned}
$$

The power transmission efficiency through the acousticelectric channel is just $\left|S_{21}\right|^{2}$, which represents the ratio of the power leaving the channel to the power entering the channel.

In order to maximize the power transmission efficiency, simultaneously conjugate impedance match for the input and output port of the channel should be performed. Sparameters can be utilized to fulfill the task. Usually a source impedance matching circuit is inserted between the power source $U_{s}$ and the input port 1 , and the load impedance

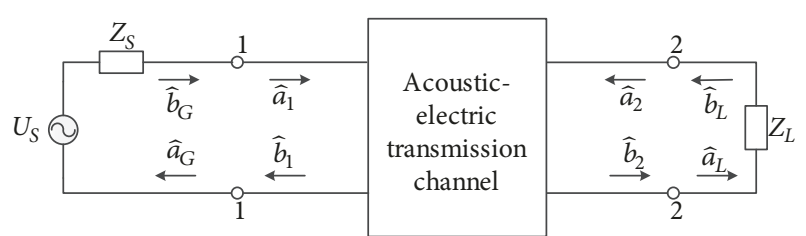

FIgURE 17: The acoustic-electric channel described by using power waves.

matching circuit is added between the output port 2 and the load $Z_{L}$. The diagram of the acoustic-electric channel with dual port simultaneous impedance matching circuits inserted is illustrated in Figure 18.

According to the principle of conjugate impedance match, there existed the following equations under simultaneously source and load impedance match.

$$
\begin{aligned}
& Z_{s}=Z_{p_{3}}^{*}, \\
& Z_{L}=Z_{p_{4}}^{*}
\end{aligned}
$$

where $Z_{p 3}, Z_{p 4}$ represent the impedance looking into ports 1 and 2 , respectively, and $*$ subscript indicates the complex conjugate operator. As $Z_{s}$ and $Z_{L}$ are known quantities, $Z_{p 3}$ and $Z_{p 4}$ are known according to (24). In order to design source and load impedance matching circuits, the impedance $Z_{p 1}$ looking into port 1 of the channel and the impedance $Z_{p 2}$ looking into port 2 of the channel should be calculated. They can be obtained based on $S$-parameters as follows:

$$
\begin{aligned}
& Z_{p 1}=\frac{1+\Gamma_{1}}{1-\Gamma_{1}} Z_{0} \\
& Z_{p 2}=\frac{1+\Gamma_{2}}{1-\Gamma_{2}} Z_{0}
\end{aligned}
$$

Here $\Gamma_{1}, \Gamma_{2}$ denote the reflection coefficient looking into ports 1 and 2 , respectively. The conjugates of $\Gamma_{1}, \Gamma_{2}$ can be calculated as

$$
\begin{gathered}
\Gamma_{1}^{*}=\frac{A_{1}-\sqrt{A_{1}^{2}-\left|2 B_{1}\right|}}{2 B_{1}} \\
\Gamma_{2}^{*}=\frac{A_{2}-\sqrt{A_{2}^{2}-\left|2 B_{2}\right|}}{2 B_{2}}
\end{gathered}
$$

where

$$
\begin{aligned}
& A_{1}=1+\left|S_{11}\right|^{2}-\left|S_{22}\right|^{2}-\left|S_{11} S_{22}-S_{12} S_{21}\right|^{2} \\
& B_{1}=S_{11}-S_{22}^{*}\left(S_{11} S_{22}-S_{12} S_{21}\right) \\
& A_{2}=1+\left|S_{22}\right|^{2}-\left|S_{11}\right|^{2}-\left|S_{11} S_{22}-S_{12} S_{21}\right|^{2} \\
& B_{2}=S_{22}-S_{11}^{*}\left(S_{11} S_{22}-S_{12} S_{21}\right)
\end{aligned}
$$

Once $Z_{p 1}, Z_{p 2}$ have been determined, we can calculate the impedance which the source and load impedance matching 


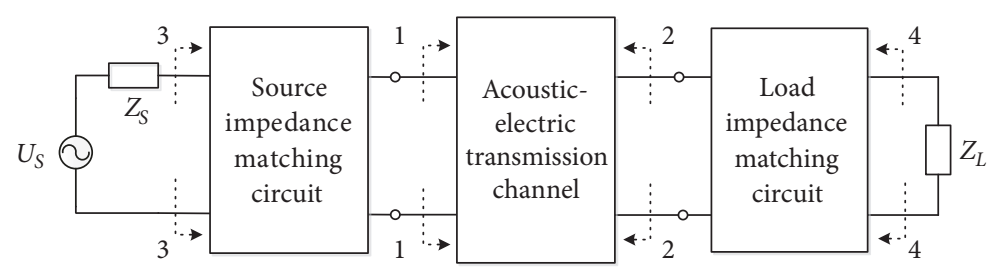

FIGURE 18: The acoustic-electric channel with simultaneous source and load ports impedance matching.

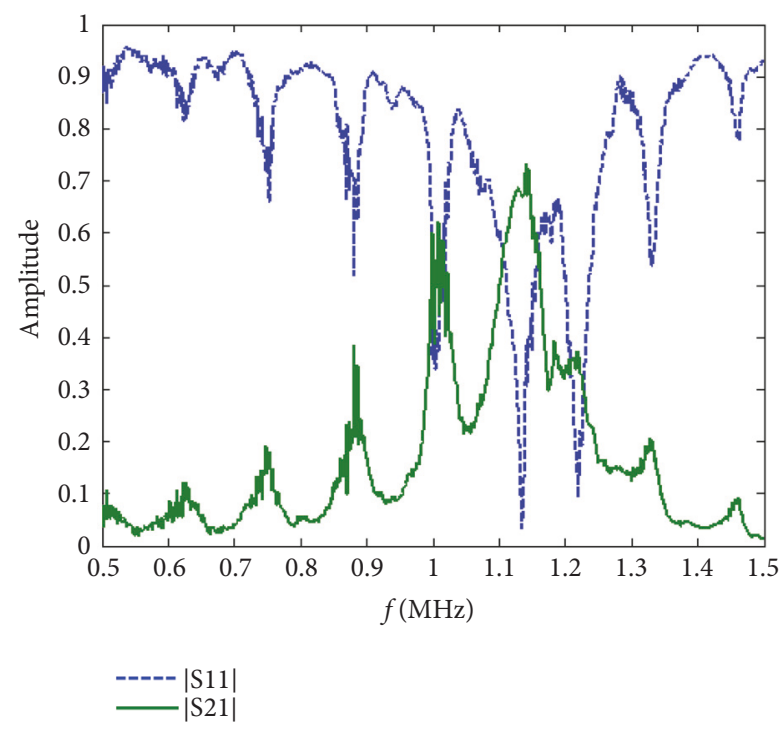

FIGURE 19: The measured amplitude of scattering parameters $S_{11}, S_{21}$ of the established channel.

circuit should compensate. Usually lumped capacitors and inductors are used to constitute the impedance matching circuit; there are several types of conjugate impedance matching circuit including $L$ type, $\pi$ type, and $\mathrm{T}$ type matching circuit for choosing [20].

As an example, we established an acoustic-electric ultrasonic power transmission channel through a planar metal wall based on APC850 PZTs with the resonance frequency of $1.1 \mathrm{MHz}$. The material properties and geometric dimensions are listed in Table 3. And the material properties of the metal barrier and epoxy are the same as that in Table 2.

The $S$-parameters of the channel are measured using DG8SAQ vector network analyzer with the reference impedance $Z_{0}=50 \Omega$. The measured amplitudes of scattering parameters $\left(S_{11}\right.$ and $\left.S_{21}\right)$ are shown in Figure 19.

It can be seen that the resonance frequency of the channel is located at near $1.1 \mathrm{MHz} . S_{11}$ represents the power wave reflection coefficient at the input port of the channel, while $S_{21}$ represents the forward power wave transmission gain of the channel. So $S_{21}$ reaches a maximum, while $S_{11}$ reaches a minimum at the resonance frequency.

The acoustic-electric channel is connected to a power source with the impedance $Z_{\mathrm{s}}$ of $50 \Omega$ and connected to a pure variable resistor as load. The load resistor is adjusted to $300 \Omega$. To maximize the power transmission efficiency, we choose the $\mathrm{L}$ type matching circuit to realize simultaneous source and load impedance matching. The diagram of the channel with impedance matching circuits inserted is shown in Figure 20. Components values of the L type impedance matching circuits are listed in Table 4.

We also measured $S$-parameters of the channel after performing source and load conjugate impedance matching. The power transmission efficiency curves versus excitation frequency with dual port conjugate impedance matching and without matching are shown in Figure 21.

The experimental measurements show that the maximum power transmission efficiency achieves a substantial improvement, up to about $56 \%$ with a $300 \Omega$ resistor load when the system realizes the simultaneous conjugate impedance matching.

The main advantage of the modeling method based on two-port network theory is that parameters describing the channel can be measured without a prior knowledge requirement. If the prior knowledge of the parts forming the acoustic-electric channel is known, the $Z, S$ and $A B C D$ parameters can also be obtained using analytical method [20]. The two-port network model of the channel can get the similar results as that using equivalent circuit method. They are essential the same in mapping the acoustic-electric channel to equivalent electrical network.

\section{Conclusion Remarks}

Systems for wireless ultrasonic power transmission through metal barriers based on piezoelectric transducers have drawn a lot of attentions because they have the advantage of maintaining structural integrity without penetration of the barriers at high power transmission efficiency. How to model the acoustic-electric channel properly is the first and critical step for optimal design of such an ultrasonic system. From discussions in preceding sections, we know that through-metal-wall ultrasonic power transmission channel can be modeled using several methods including analytical method based on piezoelectric theory and wave propagation equation, equivalent circuit modeling method, finite element modeling method, and two-port network modeling method. The tree-style taxonomy of the modeling framework for through-metal-wall acoustic-electric power transmission channels based on piezoelectric transducers is presented as shown in Figure 22.

The analytical method is based on piezoelectric theory and wave propagation equation, which is the first occurring method in literature. This method requires very complex mathematical calculations and is not practical to use for the 


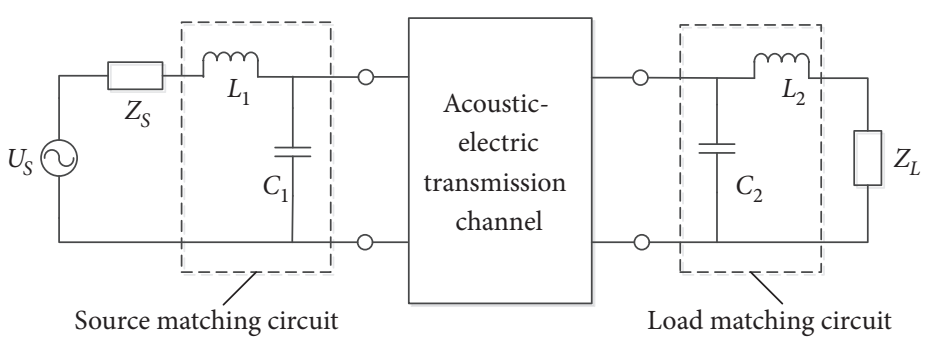

Figure 20: The diagram of the channel with L type impedance matching circuit on dual port.

TABLE 3: Material properties and geometric dimensions of APC850 piezoelectric transducer.

\begin{tabular}{cccccccc}
\hline Transducer & $d(\mathrm{~mm})$ & $l_{p}(\mathrm{~mm})$ & $\rho\left(\mathrm{kg} / \mathrm{m}^{3}\right)$ & $h_{33}(\mathrm{~V} / \mathrm{m})$ & $\varepsilon_{33}^{S}(\mathrm{~F} / \mathrm{m})$ & $e_{33}\left(\mathrm{C} / \mathrm{m}^{2}\right)$ & $Q_{m}$ \\
\hline APC850 & 25.3 & 2.05 & 7750 & $2.376 \times 10^{9}$ & $6.659 \times 10^{-9}$ & 15.82 & 80 \\
\hline
\end{tabular}

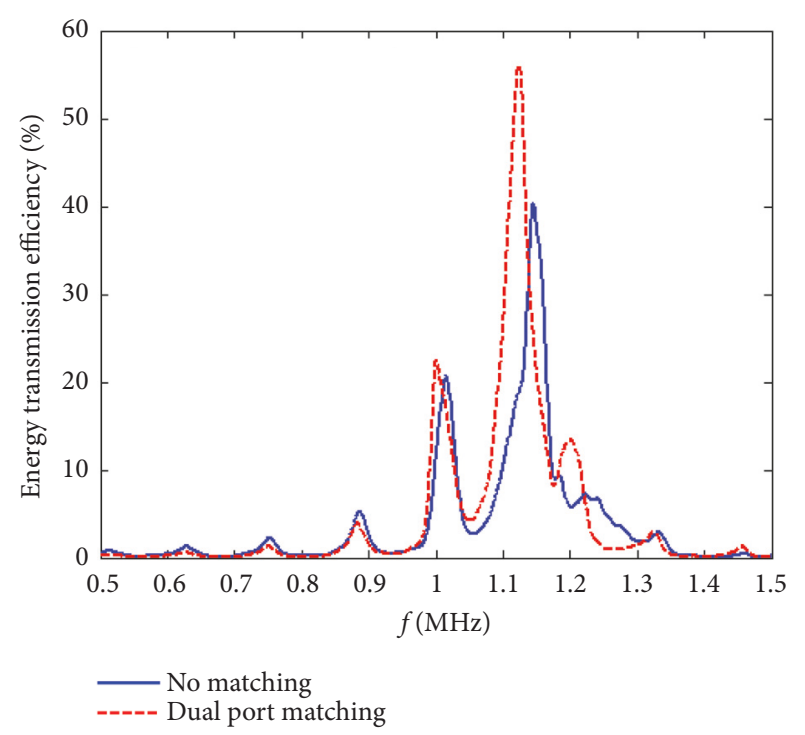

FIgURe 21: Power transmission efficiency of the channel with dual port conjugate matching and without matching.

TABLE 4: Components values of L type impedance matching circuits.

\begin{tabular}{cccccc}
\hline$Z_{\mathrm{s}}(\Omega)$ & $Z_{L}(\Omega)$ & $C_{1}(\mathrm{pF})$ & $L_{1}(\mu \mathrm{H})$ & $C_{2}(\mathrm{pF})$ & $L_{2}(\mu \mathrm{H})$ \\
\hline 50 & 300 & 430 & 10.5 & 725 & 29.6 \\
\hline
\end{tabular}

study of an ultrasonic power transmission channel with a larger number of acoustic or electronic components.

Modeling of power transmission using equivalent circuits maps the acoustic-electric channel (ultrasonic PZTs, metal wall, coupling layer, and electronic) into electric circuits by means of electromechanical analogies. This approach enables the concurrent simulation of the ultrasonic and electronic components. The two main kinds of adopted equivalent circuits are Mason's equivalent circuit and Leach's equivalent circuit. They are both effective in modeling and characterizing the channel. In the paper an ultrasonic energy transmission channel through a planar metal barrier has been established. The input impedance of the channel is simulated using Mason's circuit and Leach's equivalent circuit models and is measured experimentally. Very strong agreement between the modeled and measured data is observed, confirming the accuracy and utility of the equivalent circuit modeling methods. while Leach's equivalent circuit gets advantage over Mason's equivalent circuit for the latter can be used in circuit simulation software. So we recommend using of Leach's equivalent circuit modeling method in circuit simulations of the channel.

In consideration of metal barrier with complex geometric shape and environment conditions, a more feasible method based on finite element modeling is preferred. The experimental measurement for the impedance characteristic of a through-cylindrical-metallic-barrier channel agrees with the simulation results from the FEM model, which validate the correctness and effectiveness of FEM method. Furthermore, temperature field and acoustic field distribution in the metal wall can also be obtained. The disadvantage of FEM method is that its simulation results rely seriously on the accuracy of parameters of all parts forming the channel.

When there is a lack of the prior knowledge of parameters of the material and parts and the prototype of the ultrasonic system has been established, two-port network based modeling method is an available choice, which treats the acoustic-electric channel as a 'black box' and maps it into an electrical two-port network. The method requires much less calculation than FEM method. There are several types of network parameters. $Z$-parameters are the basic two-port network parameters, which can be converted into other types of parameters. $A B C D$ parameters are especially suitable for modeling of cascaded channels just by multiplying the $A B C D$ parameters matrix of each channel while in the range of high radio frequency, $S$-parameters are much easier to measure than $Z$-parameters. The schemes of conjugate impedance matching at both source and load port based on $S$-parameters are introduced. Experimental results show that maximum power transmission efficiency has been improved greatly, verifying the effectiveness of the simultaneous conjugate impedance based on $S$-parameters.

Though the modeling method based on a two-port network here is proposed for the acoustic-channel based 


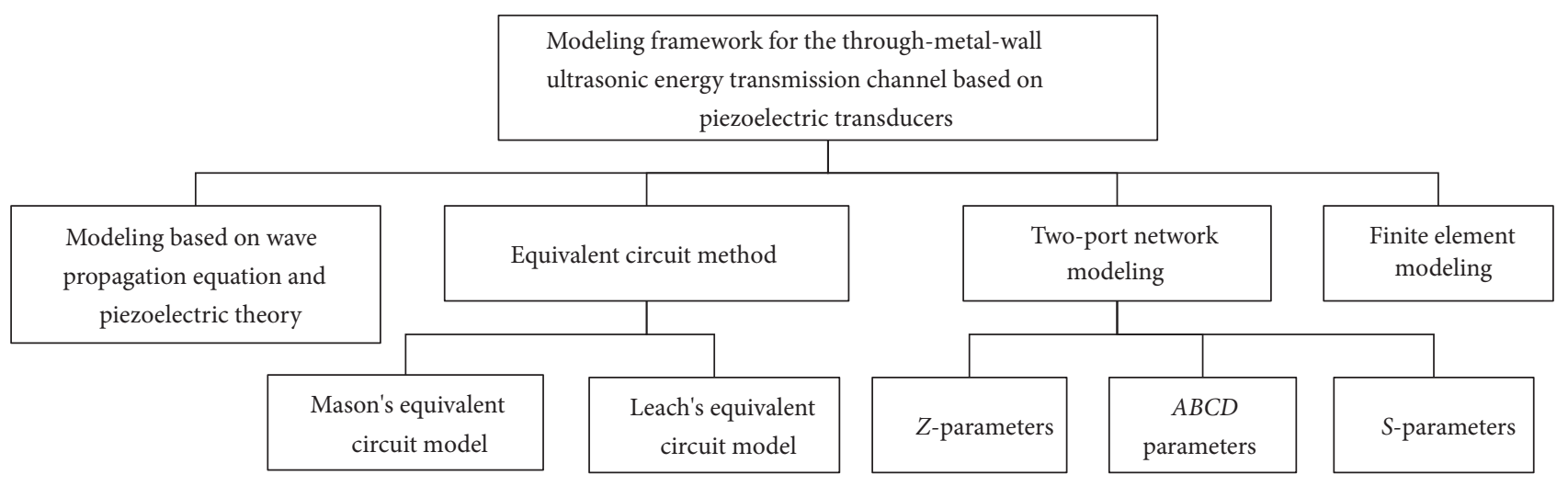

FIGURE 22: Taxonomy of the modeling framework for the through-metal-wall ultrasonic power transmission channel based on piezoelectric transducers.

on PZTs, it also can be used for modeling electromagnetic acoustic transducer (EMAT) based channels. EMATs have the advantage of not requiring direct contact with the surface of the metal barrier, which is suggested for operation on metals with surface coatings or rust [26].

As a conclusion, we present the modeling framework for through-metal-wall ultrasonic acoustic-electric channels based on piezoelectric transducers, discussing the principles of main methods, and comparing the adoptability and constraints of each method. For designing a practical system, equivalent circuit modeling method and finite element modeling method are useful for performance prediction and optimal design provided that parameters of all the parts forming the channel are known in advance. 3-dimension FEM method especially is adoptable in modeling a channel through a metal barrier with complex geometric shape. If the ultrasonic power transmission system has already been developed, two-port network parameters such as $Z$-parameters, $A B C D$ parameters, and $S$-parameters are convenient for measurement without the requirement of the prior knowledge of the channel. These network parameters can be used to characterize the channel, to perform simultaneous conjugate impedance matching to maximize the power transmission efficiency of the channel. The constructed modeling framework is hoped to be helpful to optimal design and performance prediction of through-metal-wall ultrasonic power transmission channels.

\section{Conflicts of Interest}

The authors declare that there are no conflicts of interest regarding the publication of this paper.

\section{Acknowledgments}

The authors would greatly appreciate the financial supports provided by National Natural Science Foundation of China No. 51375485 and Natural Science Foundation of Hunan Province No. 2017JJ2300 for this work.

\section{References}

[1] Y. Hu, X. Zhang, J. Yang, and Q. Jiang, "Transmitting electric energy through a metal wall by acoustic waves using piezoelectric transducers," IEEE Transactions on Ultrasonics, Ferroelectrics and Frequency Control, vol. 50, no. 7, pp. 773-781, 2003.

[2] Z. Yang, S. Guo, and J. Yang, “Transmitting electric energy through a closed elastic wall by acoustic waves and piezoelectric transducers," IEEE Transactions on Ultrasonics, Ferroelectrics and Frequency Control, vol. 55, no. 6, pp. 1380-1386, 2008.

[3] C. F. Lü, J. S. Yang, J. Wang, and W. Q. Chen, "Power transmission through a hollow cylinder by acoustic waves and piezoelectric transducers with radial polarization," Journal of Sound and Vibration, vol. 325, no. 4-5, pp. 989-999, 2009.

[4] Z. Yang, J. Yang, and Y. Hu, "Energy trapping in power transmission through an elastic plate by finite piezoelectric transducers," IEEE Transactions on Ultrasonics, Ferroelectrics and Frequency Control, vol. 55, no. 11, pp. 2493-2501, 2008.

[5] Z. T. Yang and S. H. Guo, "Energy trapping in power transmission through a circular cylindrical elastic shell by finite piezoelectric transducers," Ultrasonics, vol. 48, no. 8, pp. 716$723,2008$.

[6] H. Hu, Y. Hu, C. Chen, and J. Wang, "A system of two piezoelectric transducers and a storage circuit for wireless energy transmission through a thin metal wall," IEEE Transactions on Ultrasonics, Ferroelectrics and Frequency Control, vol. 55, no. 10, pp. 2312-2319, 2008.

[7] Z. Yang, J. Yang, and Y. Hu, "Nonlinear behavior of electric power transmission through an elastic wall by acoustic waves and piezoelectric transducers," IEEE Trans. Ultrason. Ferroelectr. Freq. Control, vol. 55, no. 11, pp. 2527-2531, 2008.

[8] S. Sherrit, M. Badescu, X. Bao, Y. Bar-Cohen, and Z. Chang, "Efficient electromechanical network models for wireless acoustic-electric feed-throughs," in Proceedings of the Smart Structures and Materials 2005 - Smart Sensor Technology and Measurement Systems, vol. 5758, pp. 362-372, March 2005.

[9] S. Sherrit, B. Doty, M. Badescu et al., "Studies of acousticelectric feed-throughs for power transmission through structures," in Proceedings of the Smart Structures and Materials: Industrial and Commercial Applications of Smart Structures Technologies, vol. 6171, p. 617102-617102-8, International Society for Optics and Photonics, San Diego, Calif, USA, 2006. 
[10] Z. Chang, X. Bao, B. J. Doty et al., "Power loss consideration in wireless piezoelectric acoustic-electric power feedthru," in Proceedings of the Sensors and Smart Structures Technologies for Civil, Mechanical, and Aerospace Systems, vol. 6529, p. 652942652942-6, March 2007.

[11] X. Bao, B. J. Doty, and S. Sherrit, "Wireless piezoelectric acoustic-electric power feedthru," in Proceedings of the Sensors and Smart Structures Technologies for Civil, Mechanical, and Aerospace Systems, vol. 6529, p. 652940-652940-7, San Diego, Calif, USA, 2007.

[12] X. Bao, L. P. Davis, B. K. Henderson et al., "High-power piezoelectric acoustic-electric power feedthru for metal walls," in Proceedings of the The 15th International Symposium on: Smart Structures and Materials \& Nondestructive Evaluation and Health Monitoring, vol. 6930, p. 69300Z-69300Z-8, San Diego, Calif, USA, 2008.

[13] S. Sherrit, X. Bao, M. Badescu, and J. Aldrich, " $1 \mathrm{~kW}$ power transmission using wireless acoustic-electric feedthrough (WAEF)," in Earth \& Space 2008: Engineering, Science, Construction, and Operations in Challenging Environments, pp. 1-10, Long Beach, Calif, USA, 2008.

[14] S. Moss, J. Skippen, M. Konak, I. Powlesland, and S. Galea, "Detachable acoustic electric feedthrough," in Proceedings of the SPIE Conf. Sensors and Smart Structures Technologies for Civil, Mechanical, and Aerospace Systems, vol. 7647, p. 764745764745-12, March 2010.

[15] S. Roa Prada, Modeling, Design and Temperature Characterization of an Ultrasonic Through-Wall Communication System, Rensselaer Polytechnic Institute, Troy, NY, USA, 2010.

[16] S. Roa-Prada, H. A. Scarton, G. J. Saulnier et al., "An ultrasonic through-wall communication (UTWC) system model," Journal of Vibration and Acoustics, vol. 135, no. 1, Article ID 011004, 2013.

[17] W. M. Leach, "Controlled-source analogous circuits and SPICE models for piezoelectric transducers," IEEE Transactions on Ultrasonics, Ferroelectrics and Frequency Control, vol. 41, no. 1, pp. 60-66, 1994.

[18] K. R. Wilt, H. A. Scarton, S. Roa-Prada et al., "Finite element modeling and simulation of a two-transducer throughwall ultrasonic communication system," in Proceedings of the 2009 ASME International Mechanical Engineering Congress and Exposition, (IMECE '09), vol. 15, pp. 579-589, Fla, USA, November 2009.

[19] K. R. Wilt, T. J. Lawry, and H. A. Scarton, "Mechanical design implications on power transfer through thick metallic barriers using piezoelectric transducers," in Proceedings of the ASME 2010 International Mechanical Engineering Congress \& Exposition, vol. 13, pp. 173-182, British Columbia, Canada, 2010.

[20] T. J. Lawry, K. R. Wilt, H. A. Scarton, and G. J. Saulnier, "Analytical modeling of a sandwiched plate piezoelectric transformerbased acoustic-electric transmission channel," IEEE Transactions on Ultrasonics, Ferroelectrics and Frequency Control, vol. 59, no. 11, pp. 2476-2486, 2012.

[21] T. J. Lawry, N. K. Dhar, P. S. Wijewarnasuriya et al., "Electrical optimization of power delivery through thick steel barriers using piezoelectric transducers," in Proceedings of the SPIE Energy Harvesting and Storage: Materials, Devices, and Applications, vol. 7683, p. 768314-768314-12, Orlando, Fla, USA, 2010.

[22] T. Lawry, A High Performance System for Wireless Transmission of Power and Data Through Solid Metal Enclosures, Rensselaer Polytechnic Institute, Troy, NY, USA, 2011.

[23] D. Yang, B. Hou, D. Tian, and S. Wang, "Modeling and characteristic analysis of wireless ultrasonic vibration energy transmission channels through planar and curved metal barriers," Shock and Vibration, vol. 2018, Article ID 5367017, 11 pages, 2018.

[24] D. A. Frickey, "Conversions between S, Z, Y, h, ABCD, and $T$ parameters which are valid for complex source and load impedances," IEEE Transactions on Microwave Theory and Techniques, vol. 42, no. 2, pp. 205-211, 1994.

[25] J. Rahola, "Power waves and conjugate matching," IEEE Transactions on Circuits and Systems II: Express Briefs, vol. 55, no. 1, pp. 92-96, 2008.

[26] D. J. Graham, J. A. Neasham, and B. S. Sharif, "Investigation of methods for data communication and power delivery through metals," IEEE Transactions on Industrial Electronics, vol. 58, no. 10, pp. 4972-4980, 2011. 


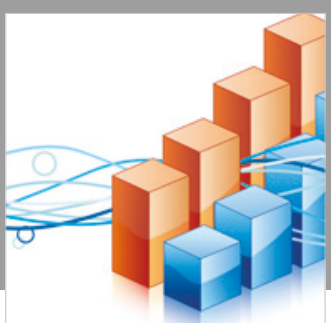

Advances in

Operations Research

\section{-n-m}
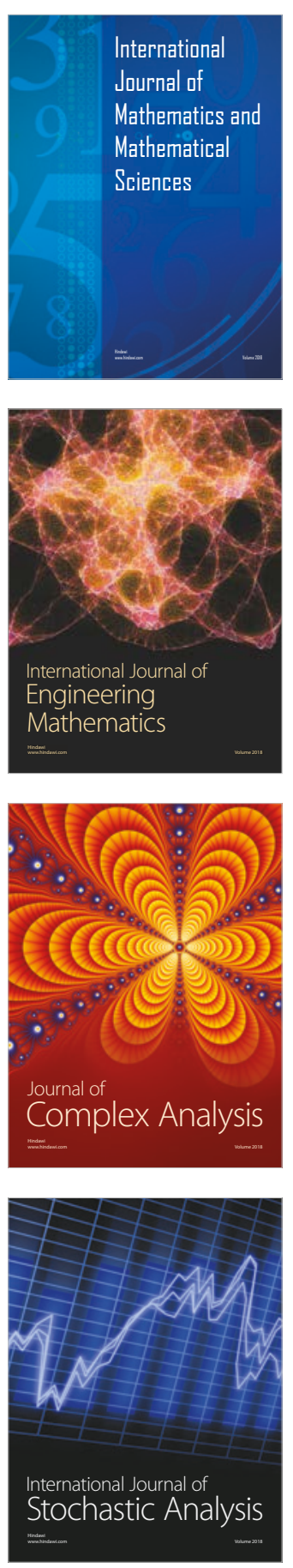
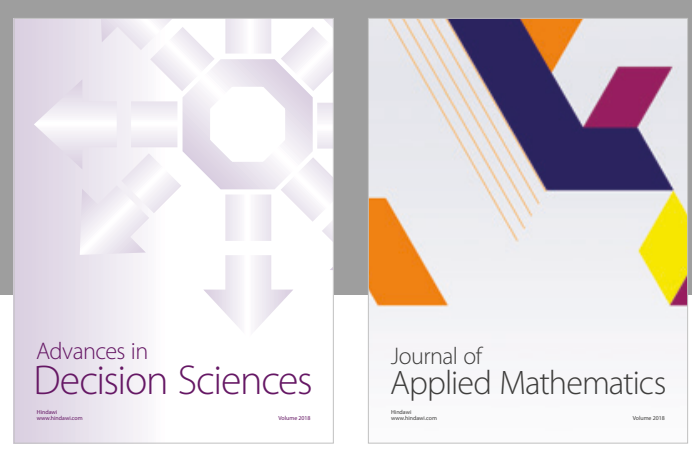

Journal of

Applied Mathematics
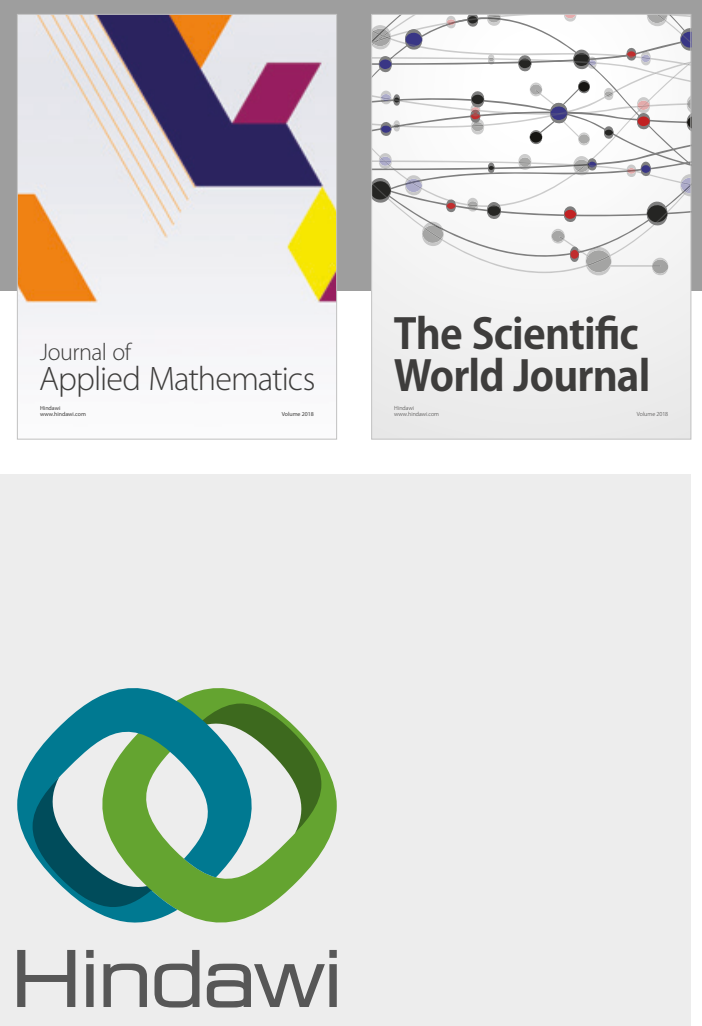

Submit your manuscripts at

www.hindawi.com

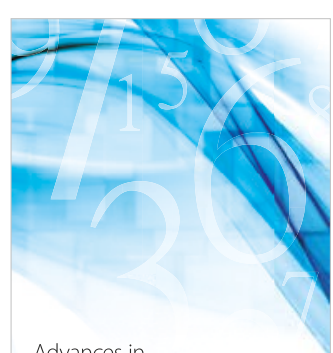

Advances in
Numerical Analysis
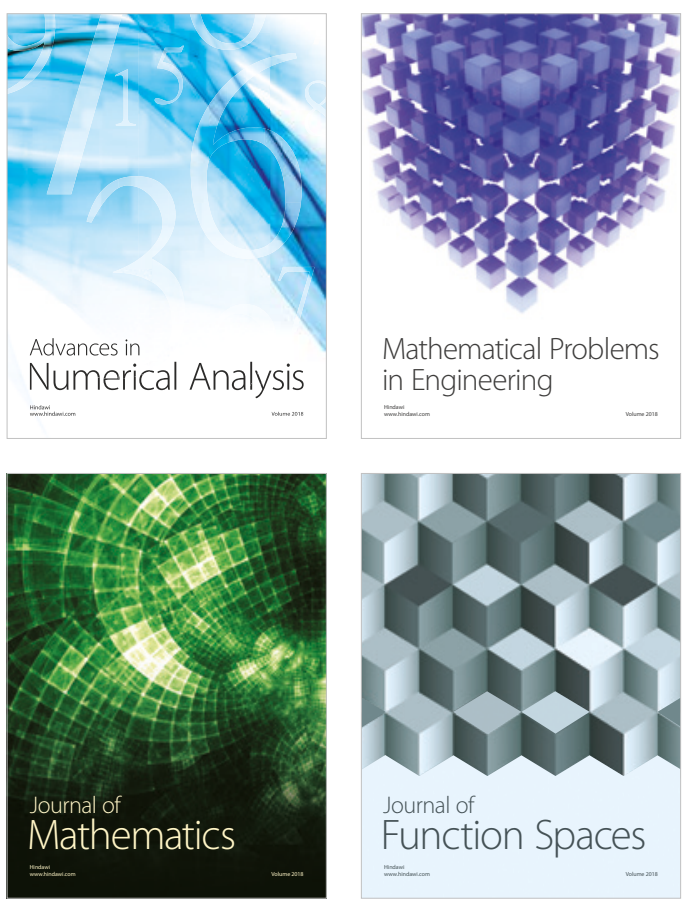

Mathematical Problems in Engineering

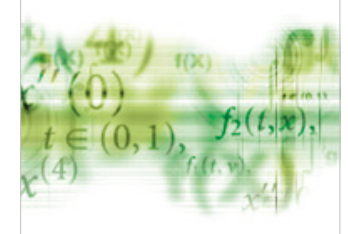

International Journal of

Differential Equations

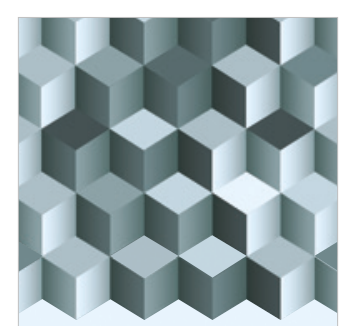

Journal of

Function Spaces

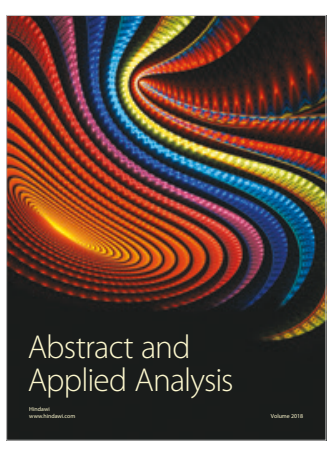

The Scientific

World Journal

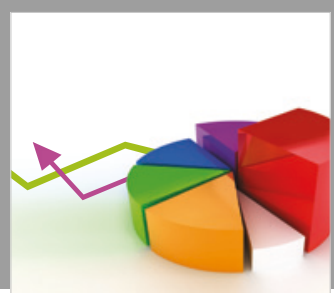

Journal of

Probability and Statistics
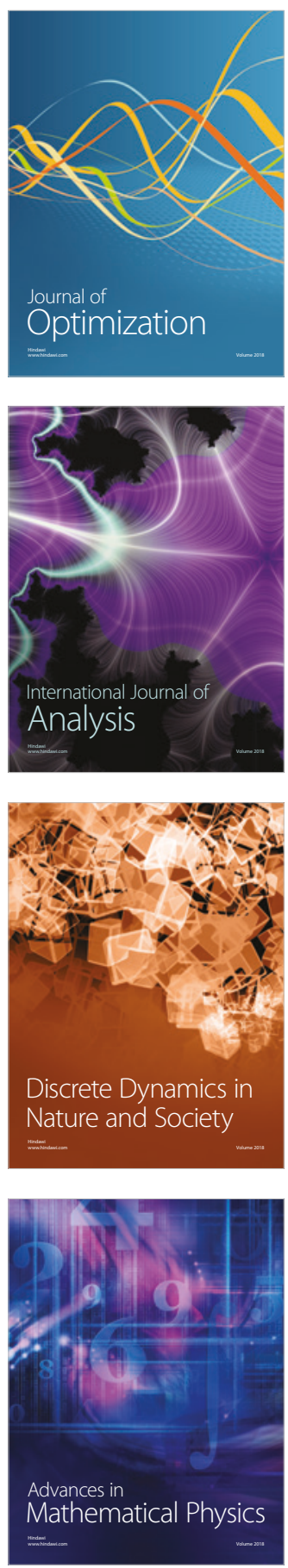